\title{
Notificações de incidentes relacionados à segurança do paciente como ferramenta de gestão para a mudança da cultura organizacional
}

\author{
Incident notifications related to patient safety as a management tool for changing the \\ organizational culture
}

Notificaciones de incidentes relacionados con paciente seguridad como una herramienta de gestión para el cambio de la cultura organizacional

Jaqueline Fátima de Souza ${ }^{1 *}$, Dagmar Willamowius Vituri².

\begin{abstract}
RESUMO
Objetivo: Avaliar as notificações de incidentes em um hospital privado de grande porte. Além de realizar a caracterização dos incidentes notificados, quanto ao turno, local e setor da ocorrência. Método: Pesquisa descritiva, exploratória, retrospectiva, de abordagem quantitativa, realizada no período de 13 de maio a 30 de junho de 2016 em um hospital terciário, filantrópica, com 350 leitos. Resultados: Os dados utilizados no estudo são secundários, originados a partir de um banco de dados institucional, de notificações eletrônicas de incidentes relacionados à assistência à saúde. O estudo evidenciou que $14 \%$ dos incidentes ocorreram dentro das UTIs, $20 \%$ do total dos incidentes estão relacionados a medicamentos, e $40 \%$ foram registradas no período noturno. Conclusão: Fica evidente que a cultura de segurança do paciente na instituição tem mostrado indícios de progressiva maturidade.
\end{abstract}

Palavras-chave: Enfermagem, Segurança do paciente, Cultura organizacional de gestão, Cuidados de saúde, Segurança.

\section{ABSTRACT}

Objective: To evaluate the incident notifications in a large private hospital. Assess the characterization of reported incidents, how to turn, location and area of occurrence. Method: Descriptive, exploratory Research, retrospective, quantitative approach, carried out during the period from 13 May to 30 June 2016 in a tertiary hospital, philanthropic, with 350 beds. Results: The data used in the study are secondary, originated from an institutional database of electronic notifications of incidents related to health care. The study showed that $14 \%$ of incidents occurred within the Icus, $20 \%$ of all incidents are related to medicines, and $40 \%$ were recorded at night. Conclusion: It is clear that the culture of patient safety in the institution has shown signs of progressive maturity.

Keywords: Nursing, Patient safety, Organizational culture of management, Health care, Safety.

\section{RESUMEN}

Objetivo: Evaluar las notificaciones de incidentes en un gran hospital privado. Evaluar la caracterización de los incidentes, cómo hacer, ubicación y área de ocurrencia. Método: Investigación descriptiva, exploratoria, enfoque cuantitativo, retrospectivo, llevado a cabo durante el período del 13 de mayo al 30 de junio de 2016 en un hospital terciario, filantrópico, con 350 camas. Resultados: Los datos utilizados en el estudio son secundarios, originado de una base de datos institucional de notificaciones electrónicas de incidentes relacionados con el cuidado de la salud. El estudio mostró que $14 \%$ de los incidentes ocurrió dentro de las $\mathrm{UCl}$, el $20 \%$ de todos los incidentes están relacionados con los medicamentos y $40 \%$ se registraron en la noche. Conclusión: Está claro que la cultura de la seguridad del paciente en la institución ha mostrado signos de progresiva madurez.

Palabras clave: Enfermería, Seguridad del paciente, Cultura organizacional de la gestión, Atención de salud, Seguridad.

\footnotetext{
${ }^{1}$ Faculdade Pitágoras, Londrina- Paraná *E-mail: jaquef.souzajp@gmail.com

${ }^{2}$ Hospital Universitário de Londrina - Paraná.
}

SUBMETIDO EM: 8/2018 | ACEITO EM: 9/2018 | PUBLICADO EM: 1/2019 


\section{INTRODUÇÃO}

A cultura institucional é o maior desafio das organizações hospitalares em prol da qualidade assistencial e, a quebra de paradigma ainda é uma barreira a ser enfrentada, principalmente ao se considerar que na área da saúde as peculiaridades do serviço e as heranças históricas desfavorecem os processos de mudança, podendo isso intervir na construção de novos valores pessoais e na prática da organização (OLIVEIRA; MATSUDA, 2016).

A mudança de cultura organizacional é um processo longo, que necessita de inúmeras ferramentas para implantar e consolidar uma gestão efetiva, considerando a estrutura, os processos e os resultados. No processo de desenvolvimento e maturidade da cultura da organização, a resistência dos colaboradores frente às mudanças pode ser um dificultador (PERTENCE; et al., 2010).

A Agencia Nacional de Vigilância Sanitária (ANVISA), desenvolveu inúmeras ações que repercutem diretamente sobre a cultura de segurança institucional como a criação da Rede de Hospitais Sentinela em 2002, que visa a disseminação do processo de gerenciamento de riscos aos hospitais, numa tentativa de melhorar a comunicação e criar uma cultura de notificação de incidentes (CASSIANI, 2010).

No Brasil foi a publicação da RDC n 36/2013, que instituiu o Programa Nacional de Segurança do Paciente e determina a obrigatoriedade da implantação do NSP (Núcleo de Segurança do Paciente) em qualquer serviço de saúde, resultado de um esforço conjunto entre a ANVISA e Organização Mundial de Saúde (OMS), numa tentativa de melhoria contínua e vigilância intensa da qualidade e segurança do atendimento à saúde (ANVISA, 2013).

Ratificando o pressuposto de que a notificação de incidentes é um passo importante para a gestão de riscos e um indicativo de maturidade da cultura de segurança institucional, autores afirmam que instituições maduras em relação à cultura organizacional reconhecem os erros e incidentes e conseguem enxergar as circunstâncias de risco. Em contrapartida, em organizações com cultura de segurança incipiente a identificação dos incidentes é prejudicada devido à subnotificação (SANTOS, 2015).

À subnotificação atribui-se alguns fatores, dentre os quais, o medo de notificar frente ao temor de exposição do notificante, que se vê fragilizado e questionado quanto à sua capacidade profissional, o que é muito comum em organizações com uma cultura de segurança incipiente (SANTOS, 2015).

Corroborando com o exposto, Magalhães (2015) afirma que os incidentes relacionados à assistência à saúde, por muito tempo, foram tratados pelos líderes como erros humanos e não erros do sistema, o que desmotiva a notificação de eventos em função do medo de que fossem utilizados para apontar e punir os colaboradores.

Nesta perspectiva, percebe-se o erro como parte da natureza humana e, portanto, existe a necessidade de se encontrar alternativas e soluções para que os incidentes não aconteçam considerando que, em sua grande maioria, os incidentes estão relacionados sistemas falhos (KOHN, 2000 e BOHOMOL, 2007).

Estudos no Brasil estimam que em torno de $60 \%$ dos incidentes ocorridos em instituições de saúde são considerados evitáveis. Cabe aqui lembrar que no Brasil ainda se convive com o problema da subnotificação relacionada à vergonha e perda do prestígio do profissional (CAMARGO, et al. 2014).

Uma gestão de riscos eficaz pressupõe a notificação como uma forma de comunicação aberta e transparente, tendo em vista a melhoria da qualidade e segurança pelo uso de ferramentas para análise dos incidentes, que subsidiam a elaboração de planos de ação para prevenção e contenção de danos é de extrema importância (CAMARGO, et al., 2014). 
Diante da relevância do desenvolvimento de uma cultura de segurança positiva em organizações de saúde é que se motivou para o desenvolvimento deste estudo, que visa avaliar as notificações de incidentes em um hospital privado de grande porte. Com seu desenvolvimento tem-se a expectativa de fornecer informações gerenciais para a tomada de decisão, possibilitando a utilização dos achados no gerenciamento dos riscos para o amadurecimento da cultura de segurança da instituição estudada. Avaliar as notificações de incidentes relacionados à atenção à saúde em um hospital privado de grande porte é o principal objetivo deste estudo, complementando com a caracterização dos incidentes notificados, quanto ao turno, local e setor da ocorrência.

\section{MÉTODOS}

Trata-se de uma pesquisa descritiva, exploratória, retrospectiva, de abordagem quantitativa, realizada no período de 13 de maio a 30 de junho de 2016 em um hospital terciário, de grande porte, de característica filantrópica, com 350 leitos, sendo 31 leitos de UTI Adulto, 10 leitos de Unidade Coronariana (UCO), 12 leitos entre UTI Neonatal e UTI Pediátrica. O sistema de atendimento de urgência e emergências é caracterizado como um sistema aberto aos serviços de atendimento móvel (SIAT e SAMU).

Os dados utilizados no estudo são secundários, originados a partir de um banco de dados institucional, de notificações eletrônicas de incidentes relacionados à assistência à saúde, referentes ao período de 01 de janeiro a 31 de dezembro de 2015. Foram coletados mediante autorização da instituição e aprovação no Comitê de Ética em Pesquisa - AEBEL (Associação Beneficente de Londrina cujo CAAE: 54613316.9.0000.5696.

Para análise estatística considerou-se as seguintes variáveis: tipo de incidente, local de ocorrência (setor), setor notificante, meses do ano, turno das ocorrências e ações imediatas. Os dados foram tabulados em planilhas eletrônicas do programa Excel for Windows e foram calculadas as frequências e percentuais.

\section{RESULTADOS E DISCUSSÃO}

No banco de dados institucional, referente às notificações de incidentes do ano de 2015, foram analisados 758 incidentes relacionados à assistência à saúde.

O referido sistema foi desenvolvido internamente pelas equipes do Setor de Qualidade e Tecnologia da Informação, a partir do contexto e cultura organizacional. Neste módulo de notificação de incidentes, o notificador pode permanecer anônimo, ou identificar-se. Nele existem campos de preenchimento obrigatório como data, tipo de ocorrência, setor notificante, setor notificado, turno das ocorrências, ações imediatas, dentre outros.

Essas notificações de incidentes, a partir da informação do setor de ocorrência, são imediatamente vinculadas ao responsável técnico deste setor. Por exemplo, se o incidente ocorreu em um setor de assistência, o enfermeiro supervisor deste setor será identificado no sistema informatizado, tendo em vista que será o responsável pelo gerenciamento de riscos desta ocorrência.

Ao Setor de Qualidade da instituição cabe o acompanhamento de todo o processo, que finaliza com o desenvolvimento de medidas para a mitigação e classificação das ocorrências conforme o nível de dano.

A partir do estabelecimento das variáveis de análise no presente estudo, as 758 notificações referentes ao ano de 2015 foram classificadas, e os dados estão apresentados nas tabelas a seguir.

Quanto ao setor que notificou a ocorrência, os dados estão na Tabela 1. 
Tabela 1- Distribuição dos incidentes segundo o setor notificante no ano 2015. Londrina-PR, 2016.

\begin{tabular}{lcc}
\hline Setores & Total & $\mathbf{( \% )}$ \\
\hline Administrativo & 1 & 0,13 \\
Anônimo & 554 & 73,09 \\
Assistencial & 2 & 0,26 \\
CCIH & 10 & 1,32 \\
Comissão Intra Hospitalar de Doação de Orgãos e & 27 & 3,56 \\
Tecidos (ClHDOTT & & \\
Cardiocat & 1 & 0,13 \\
Centro cirúrgico & 2 & 0,26 \\
Farmácia & 14 & 1,85 \\
Gerencia de enfermagem & 2 & 0,26 \\
Instituto de Hematologia & 1 & 0,13 \\
Laboratório & 7 & 0,92 \\
Manutenção & 2 & 0,26 \\
Pronto Socorro & 6 & 0,79 \\
Qualidade & 61 & 8,05 \\
Setor de internação 2 & & 4,62 \\
Setor de Internação 6 & & 3,03 \\
Setor de Internação 6B & 35 & 0,53 \\
Setor de Internação 7a & 23 & 0,53 \\
Setor de Internação 7B & 4 & 0,26 \\
\hline Total & 4 & $\mathbf{1 0 0}$ \\
\hline Fonte: Sistema informatzado da & 2 &
\end{tabular}

Fonte: Sistema informatizado da instituição.

A variável setor notificante, necessariamente não se refere ao setor em que o incidente ocorreu, pois, ele pode ter sido percebido em outra unidade assistencial. Cabe ressaltar que o sistema informatizado desenvolvido na instituição possibilita que o setor notificante possa permanecer anônimo, o que certamente interfere negativamente no gerenciamento dos riscos da notificação.

Os resultados da Tabela 1 evidenciam um alto índice de ocorrências registradas de forma anônima (73\%) fazendo supor que a cultura organizacional presente na instituição ainda não pode ser considerada positiva, pois, este anonimato leva a supor que existe o temor de que a ocorrência de um incidente seja capaz de fazer com que todo o reconhecimento do envolvido seja desconsiderado (ONA, 2015). Reafirmando o exposto, Donchin e demais autores (2012) alega que notificações de incidentes podem levar a constrangimentos e conflitos entre as equipes e colegas de trabalho, quando não se tem uma cultura de segurança positiva.

Novarettil (2013) adverte sobre a alta probabilidade de eventos adversos em Unidades de Terapia Intensiva - UTI, devido aos fatores relacionados à gravidade dos pacientes e à necessidade de decisões urgentes e de alta complexidade. Porém, de acordo com os dados da Tabela 1, nenhum incidente notificado foi realizado pelas UTIs da instituição. É possível que tenham sido notificados e categorizados como anônimos (CECCHETTO, FACHINELLI e SOUZA, 2010).

A Tabela 2, na sequência, apresenta os setores onde se atribuiu a ocorrência dos incidentes notificados no sistema informatizado da instituição. 
Tabela 2- Distribuição dos incidentes notificados em relação ao setor de ocorrência, no ano de 2015. Londrina-PR, 2016.

\begin{tabular}{lcc}
\hline Setor de ocorrência & $\mathbf{N}^{\mathbf{0}}$ & $\%$ \\
\hline Central de Material e Esterilização & 4 & 1 \\
Centro Cirúrgico & 23 & 3 \\
Diretoria técnica & 67 & 9 \\
Farmácia & 90 & 12 \\
Gerencia de enf. & 6 & 1 \\
Hotelaria & 15 & 2 \\
Internação & 23 & 3 \\
Lavanderia & 1 & 0,1 \\
Maternidade & 84 & 11 \\
Nutrição & 12 & 1,5 \\
Pediatria & 7 & 1 \\
Portaria & 1 & 0,1 \\
Pronto Socorro & 76 & 10 \\
Setor de internação cirúrgica $7^{\text {a }}$ & 16 & 2 \\
Setor de internação cirúrgica 7B & 23 & 3 \\
Setor de internação clinica 6 & & 3 \\
Setor de internação clinica 6B & 24 & 3 \\
Setor de internação clinica/ cirúrgica 2a & 20 & 3 \\
Setor de internação cirúrgica 7C & 70 & 9 \\
Unidade Coronariana & 12 & 1,5 \\
Unidade de cuidados intermediários de adultos & 34 & 4 \\
Unidade de cuidados intermediário neonatal & 8 & 1 \\
Unidade de terapia intensiva adulta 1 e 2 & 10 & 1 \\
Unidade de terapia intensiva neonatal e pediátrica & 105 & 14 \\
\hline & 27 & 3,5 \\
\hline Fonte: Sistema informatzado da instuiçä0 & 758 & 100 \\
\hline
\end{tabular}

Fonte: Sistema informatizado da instituição.

Observa-se que os setores onde se atribuiu a ocorrência de 105 (14\%) incidentes no período avaliado foram a UTI 1 e 2 . Ressalta-se que as ocorrências notificadas podem ter sido realizadas por outro setor, ou em anonimato.,

Pesquisas mostram que em UTI existe uma maior probabilidade de ocorrência de incidentes, pois estes pacientes estão mais vulneráveis aos eventos adversos em função da alta complexidade. Corroborando esta afirmação, pesquisa realizada pela Faculdade de Medicina da Universidade de Harvard evidenciou que 20\% dos pacientes admitidos em UTIs sofreram algum tipo de eventos adversos (BECCARIA, 2009).

De acordo com os dados da Tabela 2, 12\% dos incidentes notificados foram atribuídos à Farmácia, que tem como responsabilidade a dispensação de medicamentos, etapa esta de suma importância no contexto da cadeia medicamentosa. Dentre os tipos de ocorrências notificadas para o setor de Farmácia tem-se o medicamento não entregue, ou entregue com atraso.

Estes achados corroboram os resultados apresentados na Tabela 3, onde 153 notificações de incidentes foram tipificadas como erros de medicação (20\%). 
Tabela 3- Distribuição dos incidentes notificados conforme o tipo, no ano de 2015, Londrina-PR, 2016

\begin{tabular}{lccc}
\hline Tipo de ocorrência & $\mathbf{N}^{\circ}$ & $\%$ & \\
\hline Comunicação/ documentos/ internação & 50 & 7 \\
Descarte de material incorreto & 18 & 2 \\
Dieta em atraso ou não administrada & 17 & 2 \\
Erro de medicação & 153 & 20 \\
Evasão do paciente & 3 & 1 \\
Extravio de materiais e medicamentos & 23 & 3 \\
Falha no processo de exames laboratoriais & 7 & 1 \\
Flebite & 14 & 2 \\
Ocorrências que causaram danos & 18 & 2 \\
Identificação do paciente & 24 & 3 \\
Infecção hospitalar/ descumprimento de normas SCIH & 37 & 5 \\
Lesão de pele & 56 & 7 \\
Não cumprimento de fluxograma/ processos & 12 & 2 \\
Near Miss relacionado à medicação ou queda & 36 & 5 \\
Outros & 195 & 26 \\
Perda de dispositivo & 28 & 4 \\
Prescrição médica & 41 & 5 \\
Queda & 26 & 3 \\
\hline
\end{tabular}

Fonte: Sistema informatizado da instituição.

Dentre os tipos de erros de medicação encontrados no presente estudo estão a falta de medicamentos, resultando no atraso da administração. Tem-se também o erro de administração e o erro de dose e via na prescrição médica, corroborando os achados de outros estudos que atribuem a maioria dos incidentes a erros de medicação (AQUINO, 2014).

$\mathrm{Na}$ enfermagem, existe um risco elevado de incidentes relacionados a medicamentos, quem podem ser classificados desde o near miss até eventos graves, em função de que o maior número de atividades diárias realizadas pela equipe de enfermagem esta relacionada ao preparo e administração de medicamentos (NOVARETTI, 2013).

Corroborando o exposto, Padilha (1994) realizou estudos em cinco UTls de São Paulo e as iatrogênicas relacionadas a medicamentos representaram $33,4 \%$ do total.

Os incidentes envolvendo medicamentos são uma preocupação mundial e uma das prioridades dos organismos nacionais. No Brasil, dentre as diretrizes da política Nacional de Segurança do Paciente, estão o Protocolo de Segurança na Prescrição, uso e administração de medicamentos e o Protocolo de Identificação do Paciente como barreiras para mitigar estes incidentes (ANVISA, 2013).

Chama a atenção nos dados da Tabela 3, que $26 \%$ dos incidentes notificados (195) foram categorizados como "outros". Este achado sugere que os tipos de incidentes predeterminados no sistema informatizado da instituição carecem de serem revistos, tendo em vista sua ampliação, de forma aumentar a especificidade, assegurando uma gestão mais efetiva dos incidentes. 
Tabela 4- Distribuição dos incidentes segundo os meses do ano e turno de Trabalho, no ano de 2015. Londrina-PR, 2016.

\begin{tabular}{|c|c|c|c|c|c|c|c|c|}
\hline \multirow[t]{2}{*}{ Mês } & \multicolumn{2}{|c|}{ Manhã } & \multicolumn{2}{|c|}{ Tarde } & \multicolumn{2}{|c|}{ Noite } & \multicolumn{2}{|c|}{ Total } \\
\hline & $\mathbf{N}^{\circ}$ & $\%$ & $\mathrm{~N}^{\circ}$ & $\%$ & $\mathbf{N}^{\circ}$ & $\%$ & $\mathbf{N}^{\circ}$ & $\%$ \\
\hline Jan & - & - & - & - & - & - & 0 & 0 \\
\hline Fev & - & - & 1 & 100 & - & - & 1 & 0,1 \\
\hline Mar & 8 & 27 & 8 & 27 & 13 & 43 & 29 & 4 \\
\hline Abr & 12 & 22 & 6 & 11 & 38 & 68 & 56 & 7 \\
\hline Mai & 14 & 24 & 22 & 38 & 22 & 38 & 58 & 8 \\
\hline Jun & 16 & 24 & 31 & 47 & 19 & 29 & 66 & 9 \\
\hline Jul & 16 & 21 & 27 & 35 & 34 & 44 & 77 & 10 \\
\hline Ago & 9 & 24 & 12 & 32 & 17 & 45 & 38 & 5 \\
\hline Set & 21 & 31 & 12 & 18 & 35 & 51 & 68 & 9 \\
\hline Out & 30 & 28 & 45 & 42 & 31 & 30 & 106 & 14 \\
\hline Nov & 37 & 25 & 54 & 36 & 59 & 40 & 150 & 20 \\
\hline Dez & 28 & 26 & 43 & 40 & 38 & 35 & 109 & 14 \\
\hline TOTAL & 191 & 25 & 261 & 35 & 306 & 40 & 758 & 100 \\
\hline
\end{tabular}

Fonte: Sistema informatizado da instituição.

Observa-se, de forma geral, um aumento progressivo no número de notificações no sistema informatizado da instituição, o que possibilita a suposição de que a cultura de segurança do paciente na instituição encontrase em fase de desenvolvimento. Uma comunicação e efetiva são características positivas de uma cultura de segurança nas organizações de saúde para as prevenções de eventos adversos (HALLIGAN; ZECCEVIC, 2011) e o fato do número de notificações estar aumentando pode indicar que os colaboradores estão perdendo o medo de notificar, aliado ao fato de que estariam mais capacitados a reconhecer os incidentes no ambiente de trabalho.

Em relação ao turno de trabalho, a Tabela 4 mostra que ao turno noturno foi atribuído $40 \%$ dos incidentes, seguido pelo turno vespertino com $35 \%$ e matutino com $25 \%$.

Considerando que o período noturno atribui ao servidor um desgaste psicofisiológico maior do que aqueles que trabalham durante o dia em razão de que trabalham no momento em que as funções orgânicas encontram-se diminuídas (TEIXEIRA, FICHER e BORGES, 2002), é possível supor que seja este um fator contribuinte para os resultados deste estudo, porém, recomenda-se o desenvolvimento de novas pesquisas com o objetivo de estabelecer uma análise mais aprofundada sobre estes dados, estabelecendo uma correlação com o contexto da instituição em estudo. 
Tabela 5- Distribuição das ações imediatas adotadas pelo profissional envolvido, no ano de 2015. LondrinaPR, 2016.

\begin{tabular}{lcc}
\hline \multicolumn{1}{c}{ Ações Imediatas } & $\mathbf{N}^{\circ}$ & $\mathbf{\%}$ \\
\hline Ação para impedir o uso & 15 & 1,98 \\
Administrado medicação conforme prescrição médica & 48 & 6,33 \\
Aplicação de placa de hidrocolóide & 6 & 0,79 \\
Autorização para o uso apesar da manutenção da não conformidade & 1 & 0,13 \\
Comunicado laboratório para buscar o exame & 1 & 0,13 \\
Comunicado médico titular & 59 & 7,78 \\
Corrigido erro de documentação & 10 & 1,32 \\
Devolvido medicações para farmácia & 33 & 4,35 \\
Explicação / informação para o paciente & 17 & 2,24 \\
Manter em precaução de contato & 8 & 1,06 \\
Organizou o processo & 230 & 30 \\
Paciente encaminhado & 22 & 2,90 \\
Posicionado paciente novamente no leito, verificado sinais vitais & 10 & 1,32 \\
Realizado nova punção & 15 & 1,98 \\
Realizado orientações para o paciente & 8 & 1,06 \\
Realizar descarte correto do material & 9 & 1,19 \\
Realizou nova entubação & 1 & 0,13 \\
Retirado acesso venoso & 9 & 1,19 \\
Sem ação imediata registrada & 221 & 29,16 \\
Utilização de primeiros socorros & 35 & 4,62 \\
\hline
\end{tabular}

Fonte: Sistema informatizado da instituição.

Dentre as ações imediatas adotadas na instituição estudada está à revisão dos processos $(30,34 \%)$ o que está totalmente em sinergia com a lógica da Política de Segurança do Paciente preconizada pela OMS e pactuada entre os países membros da Aliança Mundial pela Segurança do Paciente (JOHNSON; GUIRGUIS; GRACE, Y., 2015). Mas, o item "sem ação imediata registrada" com $29,16 \%$ de ocorrência é um indício da necessidade de os gestores da instituição estudada adotarem estratégias para sensibilização de suas equipes quanto aos princípios do gerenciamento de riscos e segurança do paciente, dos quais se destaca a utilização dos incidentes para a elaboração de estratégias de prevenção de novos casos.

Estudos relacionados à notificação de incidentes como ferramenta de qualidade são limitados no Brasil, mesmo com a iniciativa da ANVISA, quando comparamos com outros países (ANVISA, 2013). Porém, para uma ação de melhoria e mudança de cultura organizacional, notificar é fundamental. Para isso o mapeamento de riscos é extremamente importante, pois a complexidade dos processos, com as interações sistêmicas nas unidades de saúde e o envolvimento de um número grande de profissionais, pode resultar em danos graves, irreversíveis e o óbito (GALLOTTI, 2013).

Reiterando o exposto, Silva (2011) ressalta que ao se pensar em qualidade e segurança, obrigatoriamente se deve pensar na qualificação dos profissionais de saúde, a partir de um processo de formação continuada.

A partir dos resultados encontrados neste estudo, que em sua grande maioria reiteram os achados da literatura pertinente, entende-se que é de responsabilidade de cada organização de saúde minimizar a probabilidade dos danos e incidentes aos pacientes. Adotar métodos e ferramentas de gestão da segurança baseados no princípio não punitivo, porém justo, resulta no encorajamento dos profissionais a notificar as 
ocorrências e eventos adversos, como uma forma efetiva e eficaz de criar uma cultura de segurança positiva, buscando oferecer maior segurança na assistência à saúde.

Como limitação do presente estudo tem-se a utilização de uma fonte de dados secundária, que é o banco de dados do sistema informatizado da instituição, onde a qualidade e quantidade da informação dependem do informante, repercutindo diretamente na análise dos achados e conclusões do estudo.

Como recomendação do estudo está o desenvolvimento de pesquisas futuras utilizando a busca ativa de incidentes relacionados à assistência à saúde na instituição, agregando ao atual sistema de notificação de incidentes maior potencial para a melhoria dos processos institucionais relacionados à segurança do paciente. Constata-se com o estudo que a instituição avançou significativamente no monitoramento e gestão de risco, porém, a revisão do atual sistema informatizado, por uma equipe multiprofissional, se faz necessário para o aprimoramento do processo, assim como investimentos maciços em educação permanente e continuada de suas equipes visando à transformação da cultura de segurança reativa em participativa.

\section{CONCLUSÃO}

A partir dos resultados deste estudo conclui-se que no ano de $2015,73,09 \%$ das notificações de incidentes na instituição foram anônimas e $14 \%$ dos incidentes ocorreram em UTIs. Quanto ao tipo, a maioria (26\%) foi classificada como "Outros", sugerindo a necessidade de revisão do sistema informatizado visando aumentar sua especificidade. Os erros de medicação figuraram em segundo lugar, com $20 \%$ das ocorrências. No período de estudo o número de notificações aumentou progressivamente ao longo dos meses e o turno de maior ocorrência de incidentes foi o noturno, com 40\% das notificações; e 30,34\% das ações de melhoria envolveram a organização dos processos. Fica evidente que a cultura de segurança do paciente na instituição tem mostrado indícios de progressiva maturidade, porém, esforços por parte dos gestores ainda se fazem necessários para garantir este progresso e a sustentabilidade das práticas de segurança.

Além disso, os gestores devem investir esforços no sentido de aprimorar o atual sistema informatizado de notificação de incidentes, pois, a partir da análise do banco de dados institucional percebeu-se que este sistema não prevê a coleta de informações importantes para a análise dos incidentes, apresenta algumas inconsistências no sistema de classificação da gravidade dos incidentes, além de oferecer algumas categorizações predeterminadas de variáveis que não se adequam totalmente ao contexto institucional, assim como, à taxionomia relacionada à segurança do paciente, repercutindo negativamente na análise e desenvolvimento dos planos de ação para mitigação de incidentes.

\section{REFERÊNCIAS}

1. ANVISA. Implantação do Núcleo de Segurança do Paciente em Serviços de Saúde - Série: Segurança do Paciente e Qualidade em Serviços de Saúde. Agência Nacional de Vigilância Sanitária- Brasília: ANVISA, 2013.

2. D'AQUINO FFR. Incidentes relacionados a medicamentos em uma instituição hospitalar: subsídios para a gestão. Revista Enfermagem Uerg. Botucatu. 2014. Dissertação (mestrado).

3. BOHOMOL E; RAMOS LH. Erro de medicação: importância da notificação no gerenciamento da segurança do paciente. Rev Bras Enferm; 60(1): 32-36, jan.-fev. 2007.

4. Brasil. Resolução RDC $n^{\circ} 36$, de 25 de julho de 2013 c institui ações para a segurança do paciente em serviços de saúde e dá outras providências. http://bvsms.saude.gov.br/bvs/saudelegis/anvisa/2013/rdc0036_25_07_2013.html. Acesso em: 12 março 2016.

5. CASSIANI SHB. Enfermagem e a pesquisa sobre segurança dos pacientes. Acta Paul Enferm. 2010 [acesso em 15 de julho 2012];23(6):vii-vii.

6. CAMARGO GT; QUEIROZ ALB; SILVESTRE B. et al. Cultura de la seguridad del paciente en la atención sanitaria: un estudio bibliométrico. Enferm. glob; 13(33): 336-361, ene. 2014.

7. CECCHETTO FH; FACHINELLI TS; SOUZA EN latrogrnic Or Adverse Event: Perception Of Nursing Staff. Rev Enferm UFPE on line [Internet]. 2010 jul./set [cited 2016 maio 5];4(3):1377-83. 
8. DONCHIN Y; GOPHER D; OLIN M, et al. A look into the nature and causes of human errors in the Intensive. Care Unit Crit Care Med [Internet]. 1995 [cited 2016 maio 5]; 23:294-300.

9. FICHER FM; TEIXEIRA LR; BORGES FNS; et al. Percepção de Sono: duração, qualidade e alerta em profissionais da área de enfermagem. Cad Saúde Pública, 2002.

10. GALLOTTI RMD; ASSIS SFM. Os eventos adversos em Unidade de Terapia Intensica e o Gerenciamento dos Risco as das Operadoras de Serviços. A Intersetorialidade na Gestão da Assistência a Saúde [Internet]. In: Anais do XVI Simpósio de Administração da Produção, Logística e Operações Internacionais; 2013 ago. 28 a 30; São Paulo.

11. HALLIGAN M; ZECCEVIC A. Safety culture in healthcare: a review of concepts, dimensions, measures and progress. BMJ Qual Saf. 2011; 20:338-343.

12. JOHNSON A; GUIRGUIS E; GRACE Y. Preventing medication errors in transitions of care: A patient case approach. JAPha. March-April, 2015, 55 (2):264-e276

13. MAGALÃES M. Repensando a Cultura de Segurança. Melhores Práticas em Saúde, Qualidade e Acreditação, São Paulo, Ano 3, N16, p. 40-43, ago/set/out/Nov 2015.

14. MONTERIRO C; AVELAR AFM; PEDREIRA MLG; Interrupções de atividades de enfermeiros e a segurança do paciente: revisão integrativa da literatura. Rev. Latino-Am. Enfermagem ,2015.

15. NOVARETTI MZ. Sobrecarga de trabalho da Enfermagem e incidentes e eventos adversos em pacientes internados em UTI. Revista Brasileira de Enfermagem, 2014.

16. ONA, Organização Nacional de Acreditação. Qualidade e segurança. 29 nov. 2015, 12 dec. 2015. Notas de Aula.

17. OLIVEIRA JLC; MATSUDA LM. Vantagens e dificuldades da acreditação hospitalar: A voz dos gestores da qualidade. Esc. Anna Nery Rev. Enferm; 20(1): 63-69, jan.-mar. 2016.

18. PADILHA KG. Des-cuidar: as representações sociais dos enfermeiros de UTI sobre as ocorrências iatrogênicas de enfermagem. São Paulo. Tese (doutorado) Escola de Enfemagem, Universidade de São Paulo.

19. PERTENCE PP; MALLEIRO MM. Implantação de ferramenta de gestão de qualidade em Hospital Universitário. Rev Esc Enferm USP; 44(4): 1024-1031, dez. 2010.

20. SANTOS FC. Notificar é o melhor caminho. Melhores Práticas em Saúde, Qualidade e Acreditação, Sao Paulo, Ano 3, N16, p. 46-48, ago/set/out/Nov 2015.

21. SILVA RCL; CUNHA JJA; MOREIRA CLS. Adverse Events in Intensive are: What they Know the Nurses. Revista de Pesquisa: Cuidado é Fundamental. 2001. 